\title{
Transplanted Neocortical Neurons Migrate to Repopulate Selectively Neuron-Deficient Regions After Photolytic Pyramidal Neuron Degeneration
}

\author{
Jeffrey D. Macklis \\ Department of Neurology and Program in Neuroscience, Harvard Medical School, \\ and Mental Retardation Research Center, Department of Neurology, \\ Children's Hospital, Boston, MA 02115, USA
}

The purpose of these experiments was to study neuronal migration and cellular integration following transplantation of immature neocortical neurons or pluripotent transformed neuronal precursor cell lines into a "customgenerated" pyramidal neuron deficient host. The experiments investigated both specificity of laminar positioning during cerebral development, and control mechanisms that may be manipulated for potential future neocortical transplantation. A novel model of selective neocortical degeneration using non-invasive laser illumination $/ 1 /$ was used to provide injury to callosally projecting pyramidal neurons that is geographically defined, slowly progressive, and cell-type specific. This selective neuronal injury allows precise control over the host anatomical substrate for cellular transplantation. Unfocused laser energy, at long wavelengths which penetrate through tissue without major absorption, can cause extremely selective, non-invasive damage to desired subpopulations of neurons targeted by retrograde incorporation of cytolytic chromophores which are activated by the laser energy. Intermixed neurons, glia, axons, blood vessels, and connective tissue remain intact.

Selective degeneration was effected to pyramidal neurons within neocortical lamina II/III of neonatal and young adult mice (see Figure, panel A). Embryonic day 14 or 17 (E14, E17) neocortical cell suspensions, containing recently postmitotic neurons destined to form lamina V and VI or II/III respectively, were transplanted adjacent to these geographically defined regions of ongoing neuron degeneration. Cellular injections spanned lamina II to $\mathrm{V}$, to provide donor neurons with both lateral and laminar choice for possible migration and integration. Donor cells were labeled in vitro with various combinations of unique fluorescent and electron-dense nanospheres, tritiated thymidine, and DiI, allowing distinct identification of donor cells at both light- and electron-microscopic levels. All transplanted cell suspensions were followed in tissue culture to assess labeling, viability, and differentiation following the cell preparation and stereotaxic transplantation via pulled glass micropipette.

In one set of experiments, lamina II/III neuronal degeneration was effected, E17 neurons were transplanted (neuronal birthdate destined to form these superficial layers), and experimental and control cortices were examined 1 day to 12 weeks later. Camera lucida reconstructions and higher magnification neuronal identification (by unique intracellular fluorescence within morphologically neuronal cells) were used to assess donor neuron migration and morphologic differentiation; EM was used to further confirm donor identities of migrated neurons (by incorporation of both electron dense nanospheres and a colloidal gold second label prior to transplantation). Neurons in experimental cortices migrated up to $1 \mathrm{~mm}$ and integrated specifically within the neurondeficient zones. Migration and integration did not occur in normal, unaffected deeper layers IV to VI of these experimental mice, nor in the normal lamina II/III bordering the transplantation site on the side opposite the neurondeficient region. Control grafts into intact hosts, grafts into kainic acid lesioned cortices, grafts of hypo-osmotically lysed neocortical cells, and grafts of cerebellar cell suspensions revealed 


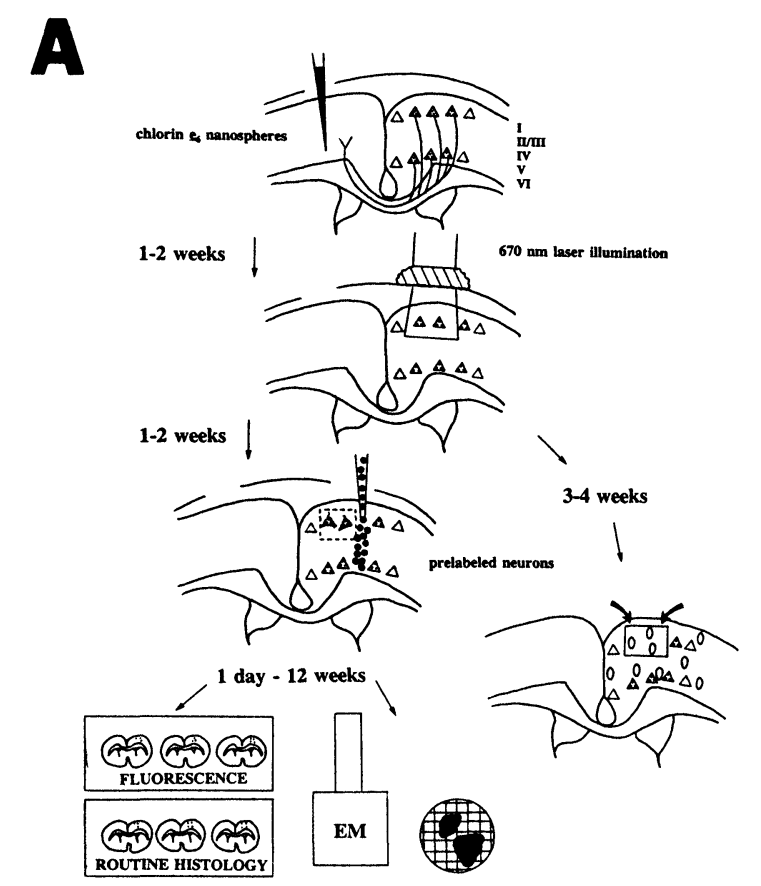

only minimal local spread without laminar preference (see Figure, panel B). Donor neurons within the lesioned zones largely assumed a pyramidal morphology and extended early processes; neurons outside these regions and in controls were morphologically non-pyramidal. By EM, donor neurons were well integrated within the surroundings, with numerous punta adherens intercellular contacts and occasional axosomatic synaptic contacts.

In a second set of experiments, transplanted E14 and E17 neurons were prelabeled in utero with tritiated thymidine during the day prior to dissociation and prelabeled with nanospheres to investigate whether E14 neurons which would normally form deep cortical layers could partially repopulate these neuron-deficient zones in superficial layers. Heavily tritium labeled neurons from both E14 and E17 transplants could be seen within the zones, developing appropriate neuronal morphology. Prelabeling with DiI in some of these transplants revealed extension of axonal processes toward

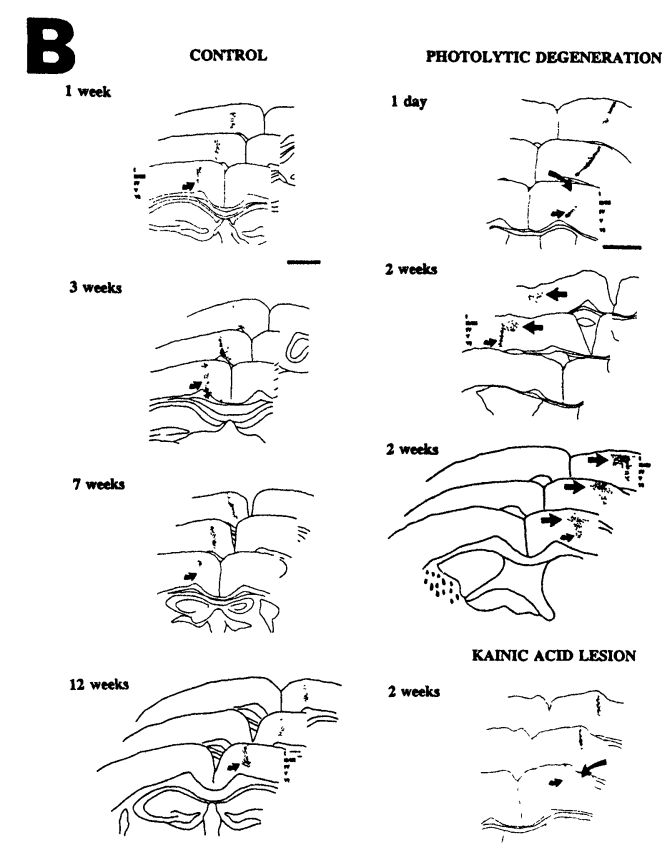

the white matter, although studies of distant projections are not yet completed. These results suggest that transplanted neocortex seeks to restore normal cytoarchitecture in these selectively neuron-deficient zones, using age-specific cues from normal development that may be reexpressed to guide migration and integration to vacant neuronal "addresses". Ongoing collaborative experiments using oncogene-transformed pluripotent hippocampal /2/ and cerebellar /3/ neural precursors that do not differentiate significantly into neurons within intact neocortex are being pursued to investigate the specificity and mechanisms of these potentially upregulated developmental signals.

\section{REFERENCES}

1. Macklis JD, Madison RD. J Neurosci 1991; 11(7): 2055-2062.

2. Renfranz PJ, Cunningham MG, McKay RDG. Cell 1991; 66: 713-729.

3. Snyder EY et al. Cell 1992; 68: 33-51. 

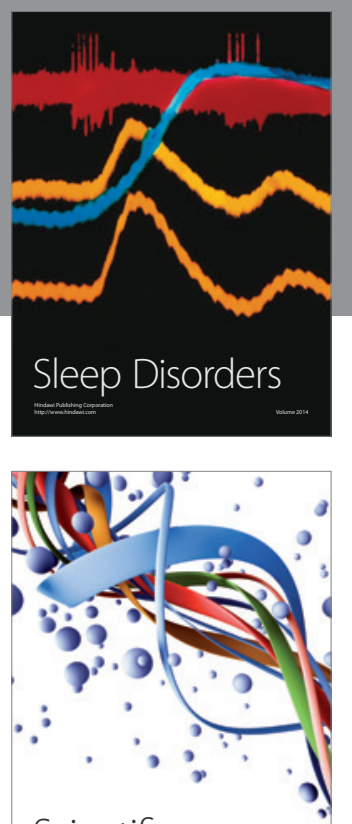

Scientifica
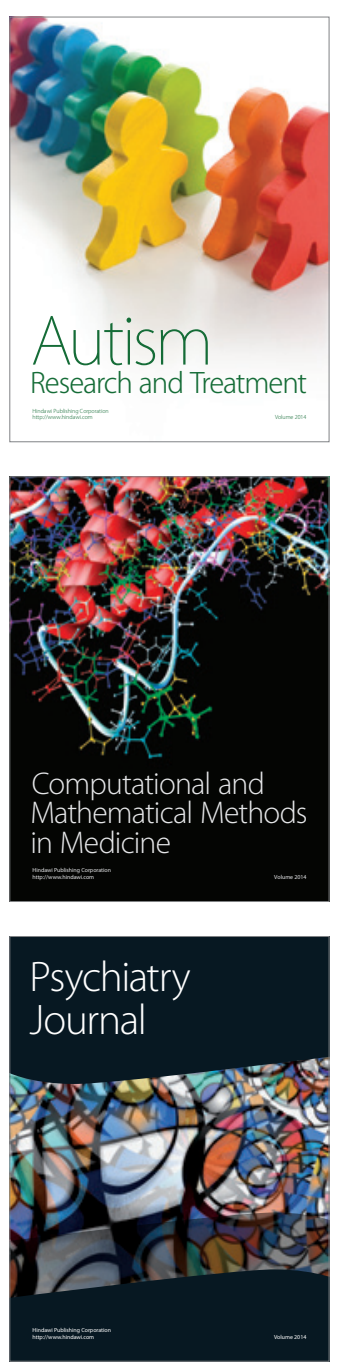
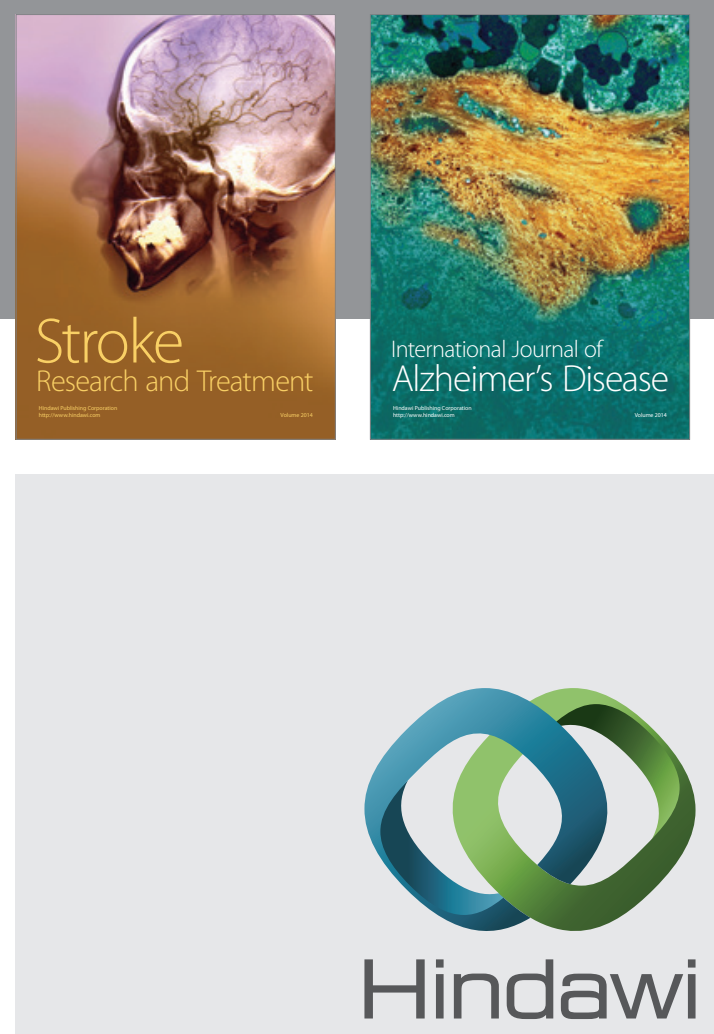

Submit your manuscripts at

http://www.hindawi.com
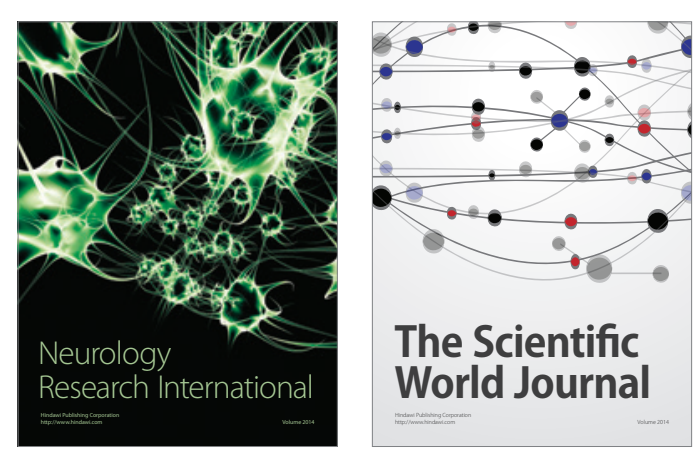

The Scientific World Journal

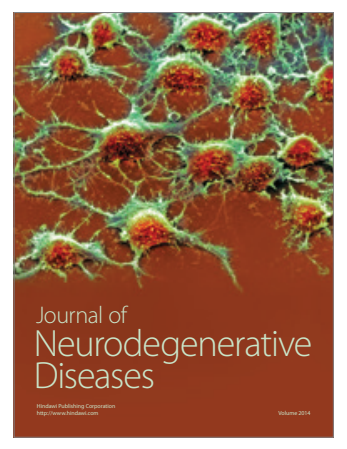

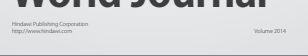

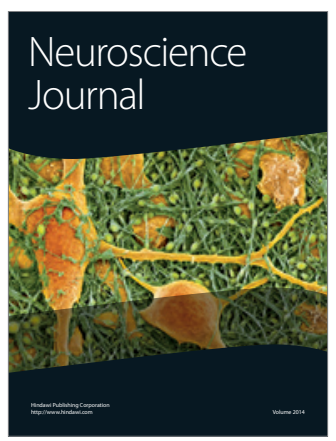

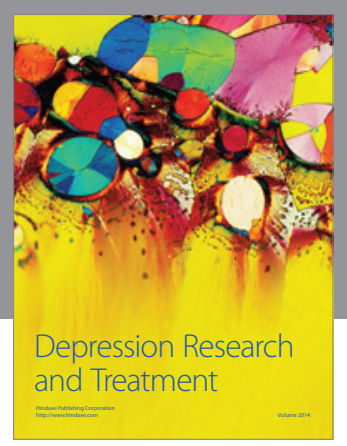
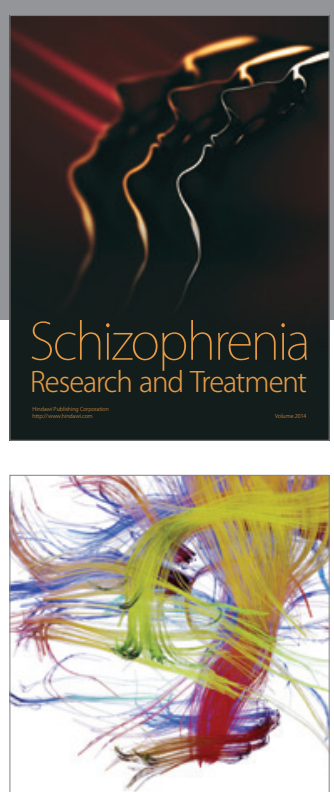

Brain Science

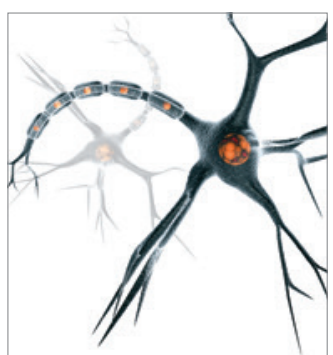

Neural Plasticity
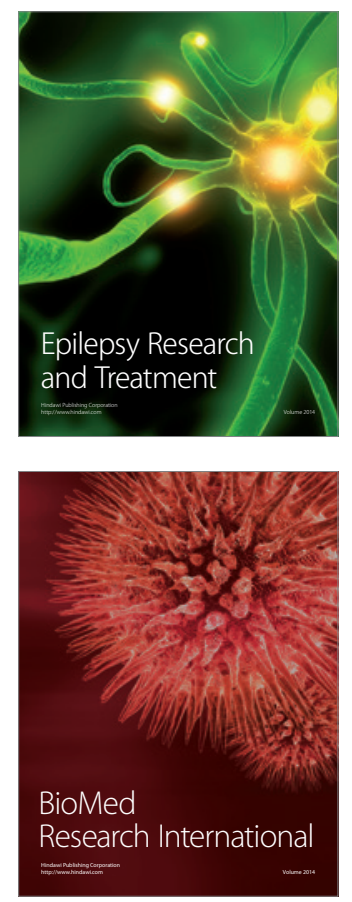

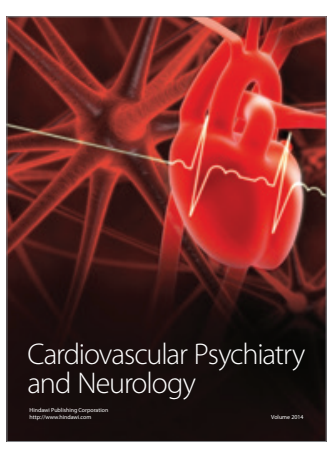

Parkinson's

Disease
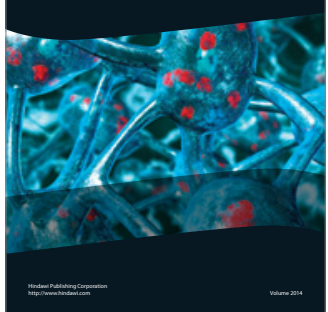